\title{
NITROGEN CONTROL OF CHLOROPLAST DEVELOPMENT AND DIFFERENTIATION
}

\section{Annual Progress Report}

\author{
Dr. Gregory W. Schmidt \\ University of Georgia Research Foundation, Inc. \\ A thens, GA 30602
}

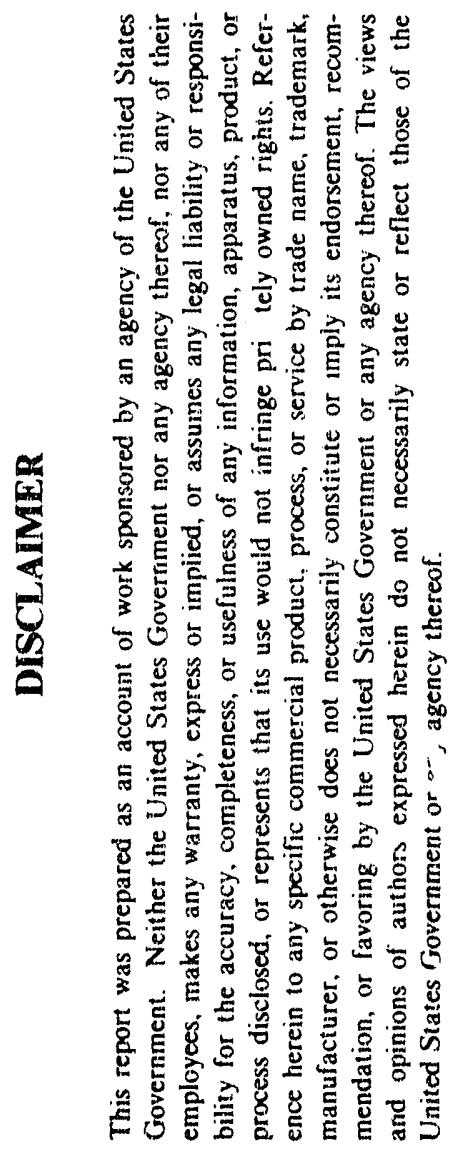

December 1991

\author{
PREPARED FOR THE \\ U. S. DEPARTMENT OF ENERGY \\ UNDER GRANT \\ DE.FG09-84ER13188
}




\section{Project Summary}

\section{Vitrosen Control of Chloroplast Development and Differentiation}

G.W. Sthmidt. Department of Botany. L'niversity of Georgia. Athens. C.A.3060)2

The sawth and development of plants and photosynthetic microorganisms is commonly limited by the aralability of nitrogen. When the synthesis of amino acids. nucleic acids and pigments is restricted by nitrogen deprivation, these organisms appear respond by altering the activities of photosynthesis and other metabolic pathways. Hence. the classical symptom for nitrogen deficiency is chlorosis. Ou: work concerris understanding the mechanisms by which plants and algae that are subjected to nitrogen jeprisition alter the composition of photosynthetic rambranes and enzymes involved in phowsynthetic carbon metabolism. Toward these ends. we study biosyntheric and gene exprossion processes in the unicellular green alga Chlamidomonas reinhardtii which is grown in an amoniumlimited continuous culture arstem. We have found that the expression of nuclear genes. including those encoding for light-harvesting proteins, are severely repressed in nitrogen-limited cells whereas. in general. chloroplast protein synthesis is attenuated primarily at the level of mR.t.t translarion. Conversely. nitrogen deprivation appears to lead to enhanced synthesis of enzymes that are involved in starch and storage lipid deposition. In addition. as a possible mearis by which photosynthetic clection transport activities and ATP synthesis is sustained during chronic periods of nitrogen depriation, thylakoid membranes become enriched with components for chlororespiration. Chatusterization of the chlororespiratory electron transport constituents. including cytochrome comprexes and $\because A D(P) H$ dehydrogenase is a major current effort. Also. we are stiving to isolate the genes encoding chlororespiration proteins toward determining how they and others that are strongly cope...ive to nutrient availability are regulated.

\section{0-1991 Progress Report and Continuation Request}

During the pas! wo years we have strived to resolve three major aspects of the effects of nirigen avalability on chloroplast biogenesis and physiology: 1) Isolation and characterization of Gnes nuclear genes that are differentially expressed as a function of nitrogen availability: : : Bucknomical and phisiological characterization of the components of the chlororespiratory electron ir.m."... pathway that is prominent in nitrogen-limied chloroplasts. if Resolution of the cunc.yences of impared synthesis of chlorophyll and or carotenoids a classical symptom of nitrogen. jeficiny on the expression of nuclear and chloroplast genes encoding pigment-binding protein. Twa - ulicitions. one of which is in review, have been generated and are appended.

The antilization of nuclear genes that are differentially expressed as a funcion of nitrogen levels ros:-2: 10 continuous cultures of Chlamdomonas entails a rather novel protocol. As outlined in he anal proposil. the small nuclear genome size of Chlamydomomas enables direor sereening w

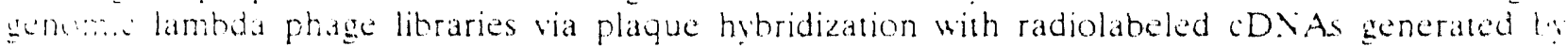
rever transcription of mRVA preparations from control vise. $\mathrm{X}$-limited cultures. Although a mult: :. ie of pliques that exhibit positive or negative control by nitrogen have heen identified and conta.....ed to be difterentially expressed. "we discovered that it was necessary to modify the procedure for frejuepuritiation and subcloning of the $13.22 \mathrm{kD}$ a inserts. This was dictated by the finding that man: i the phage harbor genes that are comstitutively expressed in addition to contiguous loci that se anogen-regulated. Hence differential hybridization signals beeome diminished as plaque purtantion proceds dese to saturation with radiolibeled. constitutive aD. A protes In these 
malances. we have resorted to amplification of phage from unpurified plaques and utilization of exise inserts for Southern biot analyses with the $\mathrm{V}$-limited and control cD. As. Lpon identification of the gene fragments that are differentially expressed. preparative agarose gel electrophoreses is employed for isolation of the DNA of interest and its subcloning into plasmids. These. together with larget clones that have been successfully obtained through plaque purification. are then used to reatlirm gene copy number in Souther blots, to characterize the transcription products detected in northern blots, and finally to assess the nature the gene product and its possihle regulatory features bi sequencing. The last aspect of this project has just been initiated and will he a major effort during the corthooming funding period.

As established in the accompanying reprint. a somewhat surprising characteristic of nitrogenlimiled ceils is the high activity of chlororespiration and the coincidental high ahundance of $\therefore$ AD (P)H dehydrogenase and novel cytochromes ( $h 1$ and h. ) associated with thylakoid memtranes. $1 \mathrm{C}$ have proposed that the development of this activity enables the maintenance of the photosynthetic electron transport chain under conditions when $\mathrm{CO}_{2}$ reduction might be (or need to he alienuated. Thus. nitrogen-limited chloroplasts become somewhat distended with starch and rrglyeride deposits when cell growth and division is restricted by limitations of amino acids and nucleotide. There are several outstanding problems about chlororespiration that include: 1) Is the pathway requisite for prevention of sundry photoinhibitory reactions and. hence. cell survival? 2) What are the biochemical teatures of the chlororespiratory electron transport chain with regard to the jehyotogenase. putative cytochrome he complex and cytochrome oxidase? 3 ) Where are the s. humis of chlororespirator electron transport encoded?

For problem 1. it will be necessary to generate mutants with impaired chlororespiratory A.sity and lest for their ability to surve nitrogen deficiency. Aiternatively. specific inhibitors of choruespiration might be employed but the best of these. antimycin and myothiazol are also inhitilors of mitochondrial respiration. Moreover since we control the extent of nitrogen deficiency trrough the use of a comtinuous culture stxtem. the inhibitor approach would he quite costly. Hence. the mutant approach is more appropriate if useful strains can be selected. We have ohtained one

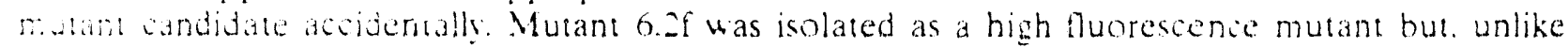
aher strins of this phenorype no defeets in photosynthetic elactron transport could be disorned.

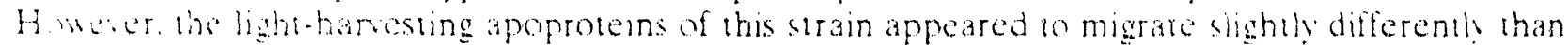

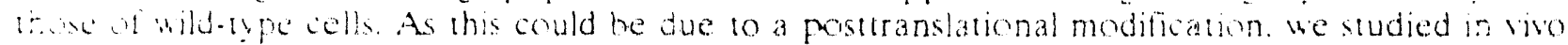

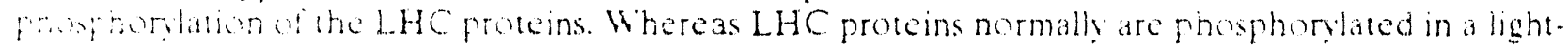
Seaten manner those of 6 ar are phosphoryated also in darkness. We reasoned ihis could be due

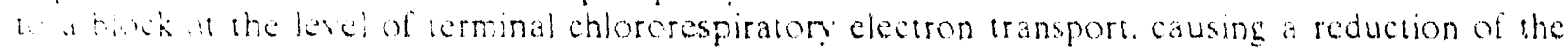

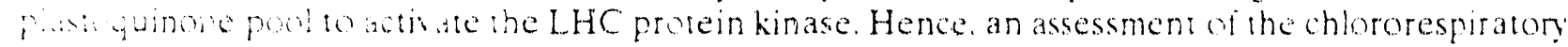

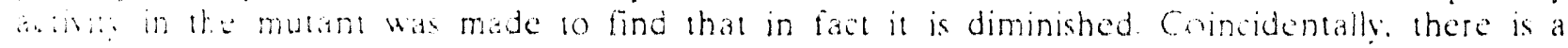

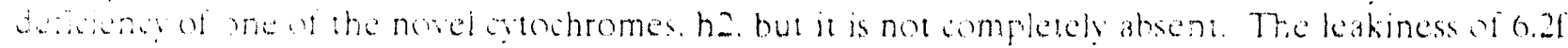

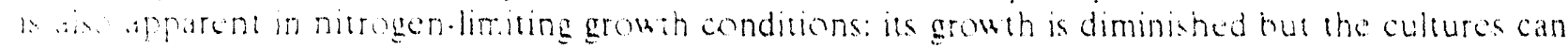

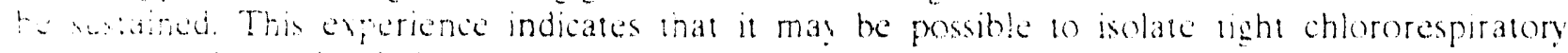

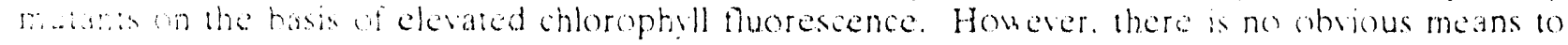

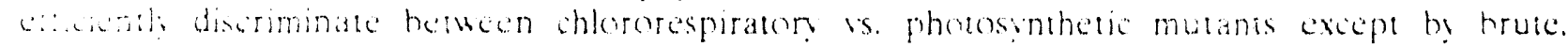

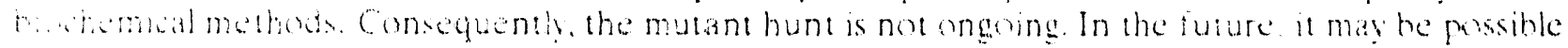

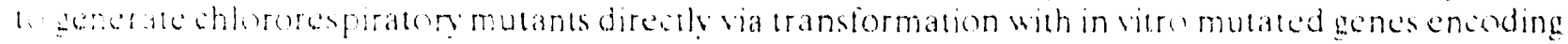

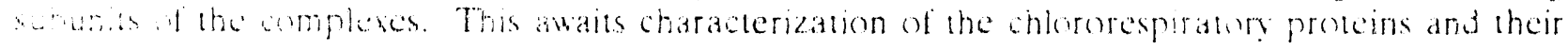
sctic 
A second vear graduate, Jelf Blanchard, has embarked upon purification of the

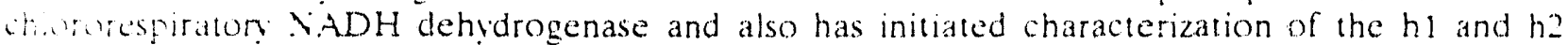
andromes. With non-ionic detergents. solubilized thylakoids from N-limited cells yield membrane Whipeses shat have been fractionated by sucrose gradient centrifugation. Cytochromes hl and h2 cosciment as high-molecular weight complexes that pellet with Photosvistem I particles. The whatrogenase is of smaller size and co-sediments with the residual light-haresting complexes of $y$. limited thylakoids. Although these complexes are present in low yields from control cells grown minsophicalis: we have decided that an expedient means to purify the complexes away from phoninthetic contaminants is through the use of Chlambdomonas mutants that are missing LHC. PSi if hoth. Consequently. it should be possible to obtain intact complexes suitable for spectroscopic an inses and protein purification. An alternative method is to employ affinity chromatography. with immotilized cyochrome $\mathrm{c}$ to enrich for cytochrome oxidase. Finally, we have obtained or requested antibo bies ro subunits of atochrome oxidase. some of which have been shoun to cross-react with the coresponding proteins of bacteria and cyanobacteria. These can be used for inmunoaffinity puritiztion as well as measurements of immunological relatedness via immunoblotting. We have repred that the major subunits of the dehydrogerase have molecular sizes that are close to that of Unanocteria (Peltier. G. and G.W. Schmidt. 1991. Proc. Natl. Acad. Sci. LSA. 88:4791-4795) and he:se. we expect the chiororespiratory components to be structurally related to the complex of the prosinutic plasma nembrane. Following purification of the major subunits. they will be subjected

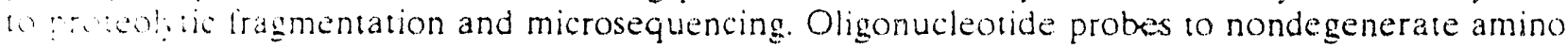
11:- sogueaces will be employed to screen the genomic lambda library and differentially regulated guas that have then described above.

The final problem we have assessed. the roles of pigment synthesis in the expression of pig....r.ibinding proteins, has been completed and is described in the accompanying preprint. We

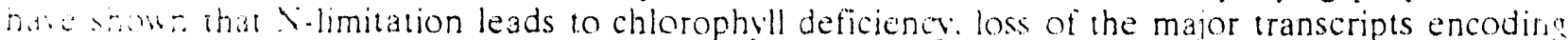
the a.sere-encoded LHC apoproteins and an apparent attenuation of the translation of chloroplast

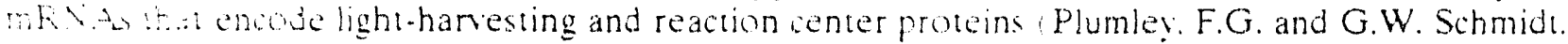

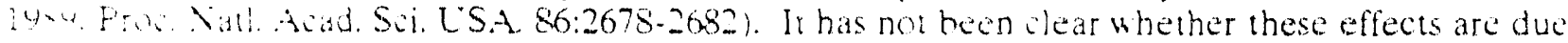

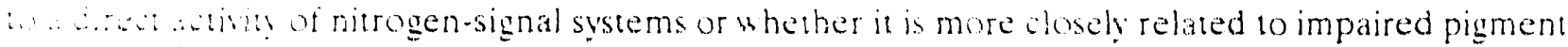

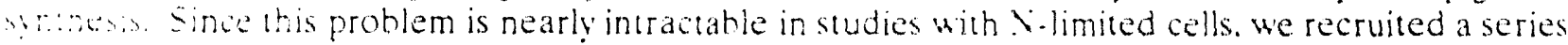
$\therefore$;.... m....munhis mutants that are blocked in chlorophyll or carotenoid synthesis. In addition. the m. anc... of light in the expression of the genes encoding these proteins was examined. It is h.... - it.. i regulation of nuclear LHC gene expression and shloroplast mRNA translation are not atfuse. by pigment synthesis in the alga since normal levels of the transeriots are present and normat rate : anroplast protein synthesis are obtained. However. both chlorophyll and carotenoid are r......... : statization of the chloroplasteneoded proteins, which when synthesized in their sta.... a. degraded following thyiakoid insertion. It also appears degradation is a major means of

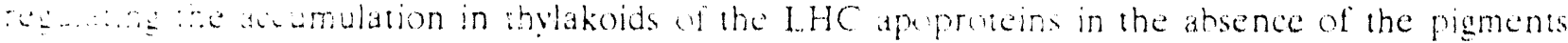

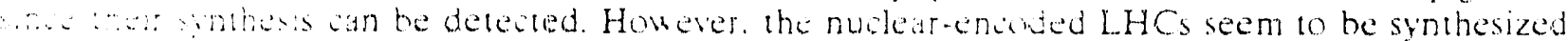
$\therefore$ ances rase rasing the possibility of translational controls elicired by pigment-deficiency. Tr............ regutation is even more conspicuous in dark-grown. chlorophyll-less ceils but under and a a sums the level of LHC transcripts is aiso altered in a manner similar to but less severe

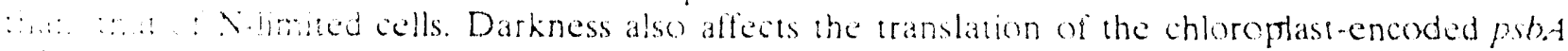
aks $A$ Heser. this effect is likely due to differences in the rate of selective turnover of this

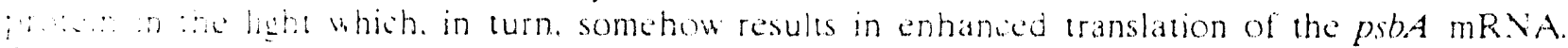

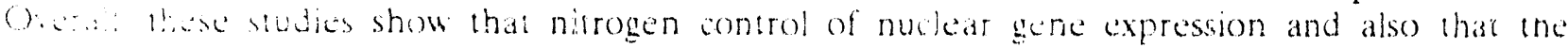

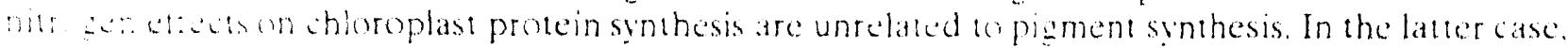


$N$-limitation, seems to affect chloroplast protein synthesis at the translational, as opposed to the posttranslational, level. 

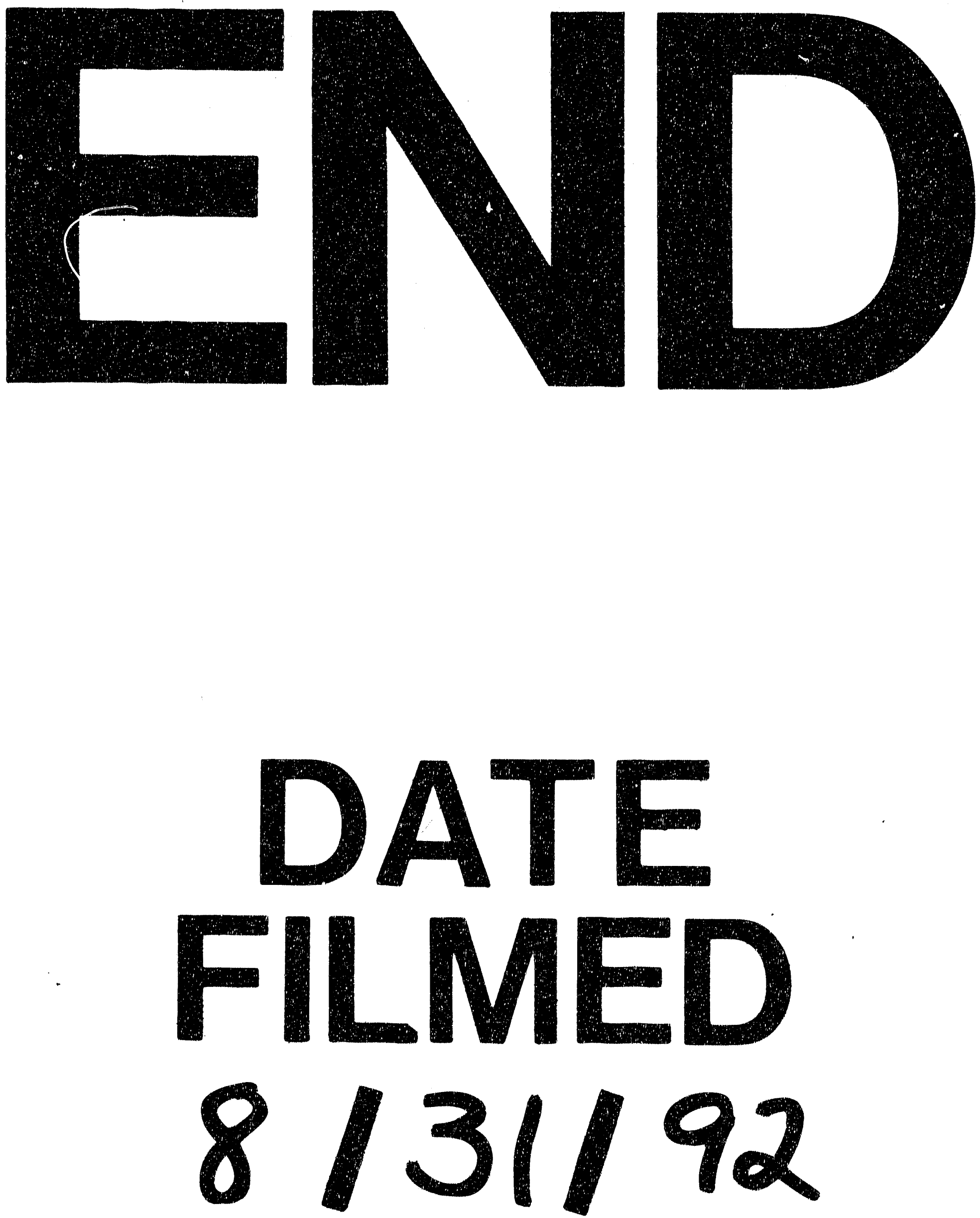
